\title{
Die Komplementärmedizin in der Onkologie - schwieriger Anspruch an Wirksamkeit, Qualität und Wirtschaftlichkeit
}

\author{
Dieter Melchart
}

Kompetenzzentrum für Komplementärmedizin und Naturheilkunde (KoKoNat), Klinikum rechts der Isar, Technische Universität München, München, Deutschland; Institut für Naturheilkunde und integrative Medizin, UniversitätsSpital Zürich, Zürich, Schweiz

Die gesamte Medizin ist seit Jahren mit steigenden Forderungen nach mehr Evidenz und Qualitätssicherung konfrontiert. Von dieser Entwicklung wird die sogenannte Komplementärmedizin in der Onkologie in besonderer Weise berührt. Nur: Wer soll diese Aufgabe lösen, und wie? Die Komplementärmedizin hat ein erhebliches Defizit an kontrollierten klinischen Studien, und es existieren derzeit kaum Fachgesellschaften und Lehrstuhlstrukturen, die für dieses Anwendungsgebiet eine ausreichende Koordinationsund Leitkompetenz besitzen. Daher sind die einzelnen Arbeitsgruppen für Komplementärmedizin in der Onkologie aufgefordert, einen eigenen Weg für eine systematische und kritische Evaluation wie Qualitätssicherung zu finden. Dabei ist die vermehrte Durchführung von evidenzgenerierenden klinischen Studien als auch eine alltagsnahe und anwenderorientierte Dokumentation der Versorgungswirklichkeit zu berücksichtigen.

Es wäre wünschenswert, wenn sich neben der Deutschen Krebshilfe mit ihrer vorbildlichen Projektförderung des Kompetenznetzes Komplementärmedizin in der Onkologie (KOKON) [1] auch staatliche Fördereinrichtungen mit neuen Forschungsprogrammen an dieser Aufgabe beteiligen würden.

Das Interesse an dieser Art von Forschung ist jedoch in unserer Gesellschaft sehr unterschiedlich verteilt: Universitäre Vertreter der Komplementärmedizin sind vorwiegend an den Fragen der wissenschaftlichen Evidenz interessiert, Krankenkassen orientieren sich an Wirtschaftlichkeit und Versichertenwettbewerb und Praxisärzte sowie Kliniken an Patientenanbindung und positiver Außendarstellung. Patienten und zuweisende Ärzte benötigen Informationen für die Inanspruchnahme komplementärer Behandlungsmethoden - normative Instanzen wie z.B. das Bundesamt für Gesundheit entscheiden vorwiegend auf der Grundlage von Wirksamkeitsnachweisen im Sinne der evidenzbasierten Medizin.
Die Prüfung einzelner Bausteine in randomisierten Studien wäre wünschenswert, ist aber bei den meist sehr individualisierten und klinik- und praxisspezifischen Vorgehensweisen oft nicht repräsentativ und nur von begrenzter Aussagefähigkeit. Ein wissenschaftlicher Wirksamkeitsnachweis im engeren Sinne ist deshalb für die in der Naturheilkunde und Komplementärmedizin sehr unterschiedlichen und im zeitlichen Verlauf variablen «Therapiecocktails» kaum zu erbringen. Meist fehlt die für eine Verallgemeinerung notwendige Repräsentativität und Reproduzierbarkeit der Methode: Pflanzenextrakte enthalten häufig keine standardisierten Inhalte, Akupunkturbehandlungen werden in Form verschiedenster Techniken, Schulmeinungen und persönlicher Therapeutenqualifikation ausgeübt und als Ergänzung oder Alternative zur konventionellen Therapie durchgeführt. Die randomisierte Vergleichsstudie kann deshalb im Bereich von Naturheilverfahren/ Komplementärmedizin nicht immer der erste Schritt einer klinischen Forschungsaktivität sein, da häufig andere Fragen als die Wirksamkeit eines einzelnen Behandlungsverfahrens im Vordergrund der Gesamtproblematik stehen oder das methodische Design für die Fragestellung nicht geeignet ist. Dies liegt an den zum Teil prinzipiellen Unterschieden, die zwischen der an der Arzneimittelforschung orientierten Forschungsstrategie und der naturheilkundlichen Wirksamkeitsprüfung bestehen. Bis ein Arzneimittel für die medizinische Versorgung empfohlen und zugelassen werden kann, sind die bekannten Phasen der klinischen Prüfung zu durchlaufen. Die Innovation gelangt also nur in die Versorgung, wenn ausreichende Evidenz durch Studienergebnisse vorliegt. Insbesondere in der komplementärmedizinischen Onkologie besteht bereits eine hohe Migration des «Produktes» Naturheilverfahren in die medizinische Versorgung, aber eine niedrige bis fehlende Studienevidenz. Die Tatsache, dass Naturheilverfahren und andere traditionelle Heilsysteme bereits vor einem potenziellen Evidenz-

\section{KARGER}

Fax +497614520714
() 2015 S. Karger GmbH, Freiburg

$1661-4119 / 15 / 0221-0008 \$ 39.50 / 0$
Univ.-Prof. Dr. Dieter Melchart

Kompetenzzentrum für Komplementärmedizin und Naturheilkunde (KoKoNat)

Klinikum rechts der Isar, Technische Universität München

Kaiserstr. 9/Rückgebäude, 80801 München, Deutschland

dieter.melchart@lrz.tu-muenchen.de 
nachweis für Ärzte und Patienten verfügbar sind, hat zur Folge, dass primär praxisrelevante Fragen an die Forschung gestellt werden und seitens der Patienten, Leistungsanbieter, Politiker und der Versicherungswirtschaft ein hoher Bedarf an Informationen über die Behandlungsqualität besteht.

Die Arbeitsgruppe «Komplementärmedizin in der Onkologie» des Tumorzentrums München (TZM) sieht deshalb im Aufbau eines Kasuistik-Archivs eine erste und machbare vertrauensbildende Maßnahme. Durch eine kontinuierliche und systematische Erfassung von Interventionen und deren Ergebnissen bei einzelnen Tumorpatienten soll mithilfe eines webbasierten Dokumentationssystems in einigen komplementärmedizinischen Praxen und onkologischen Kliniken die Anwendung und Auswirkung von Interventionen in der klinischen Routine bzw. im ärztlichen Alltag abgebildet und die typische Patientenklientel dargestellt werden. Dieser resultatorientierte Ansatz beschreibt medizinisch definierbare Ergebnisse wie Befund- oder Symptomänderungen, Stress- und Fatigueabbau sowie verschiedene gesundheitsorientierte Indikatoren wie Schlaf, Abwehr, Thermoregulation (körperliche Basisfunktionen), Selbstwirksamkeit, Entspannungsfähigkeit und emotionale Selbstregulation (kognitiv-psychologische Basiskompetenzen). Weitere Aspekte sind Sicherheit und Kostenerfassung.

Als nächster Schritt ist die Umsetzung eines naturheilkundlichen Patienten-Kompetenztrainings in Form eines Nebenwirkungsmanagements mit Selbsthilfetechniken vorgesehen [2]. $\mathrm{Zu}$ einem späteren Zeitpunkt soll dann entschieden werden, welche Maßnahmenbereiche durch randomisierte kontrollierte Studien evaluiert und mithilfe des TZM (hoffentlich) in eine Forschungsförderung eingebracht werden können.

Bei Interesse an aktiver Beteiligung am Netzwerk steht der Autor gerne als Ansprechpartner zur Verfügung.

\section{Literatur}

www.kompetenznetz-kokon.de (Zugriff 27.02.2015).

2 Melchart D, Bohnes E, Doerfler W, Eustachi A, Herrmann T, Wellenhofer-Li Y: Kompetenztraining für Patienten. Deutsche Zeitschrift für Onkologie 2014;46:158-162. 